\title{
Development and analysis of AIDS epidemic agent-based computer model applying an algorithm for explicit calculation of HIV replicability
}

\author{
Anna Smirnova \\ ICG SB RAS, Novosibirsk, Russia \\ NSU, Novosibirsk, Russia \\ asmirnova@bionet.nsc.ru
}

\author{
Mikhail Ponomarenko \\ ICG SB RAS, Novosibirsk, Russia \\ pon@bionet.nsc.ru
}

\author{
Sergey Lashin \\ ICG SB RAS, Novosibirsk, Russia \\ NSU, Novosibirsk, Russia \\ lashin@bionet.nsc.ru
}

\begin{abstract}
Different strains of HIV contribute differently to the course of the disease. For its evaluation, 1,336 HIV strains were analyzed. An agent model of the spread of HIV infection in the population has been developed. We analyzed 5 scenarios for the development of the HIV epidemic in Russia, depending on the initial data. Without additional measures, after 10 years, the percentage of HIV patients in Russia will increase from $1 \%$ to $2.45 \%$. Comprehensive measures to increase the use of barrier contraception, reduce the number of joint injections among drug users and improve the situation with treatment coverage can reduce the percentage of patients from $1 \%$ to $0.3 \%$ and prevent the emergence of new patients.
\end{abstract}

Keywords - agent-based model, prediction, simulation, TBP/TATA, HIV, AIDS

\section{MOTIVATION AND AIM}

The AIDS epidemic is an extreme problem that is relevant not only for Russia, but also for the whole world. According to statistics, about $1 \%$ of the Russian population is HIV-infected. HIV has a large number of genetic variants that differ in both replication and virulence. The duration of the incubation period and the severity of the disease in the active phase also depend on these factors. Currently, a large number of mathematical and computer models of HIV infection have been created that consider infection at different levels of biological organization: from populationepidemiological to molecular-genetic. However, in the modern literature, we have not encountered models that combine the genetic and population-ecological levels.

\section{METHODS}

The statistical method [1] of computer prediction of the affinity of the TBP/TATA complex was used to assess the replication and virulence of various genetic variants of HIV. The Python programming language was used to process the HIV strain database and visualize the data. MAFFT and BioPython were used to build a phylogenetic tree. The agent model of the AIDS epidemic is developed in the $\mathrm{C}++$ programming language.

\section{RESULTS}

The first step was a genetic analysis of the HIV strains obtained from the NCBI database. Based on the analysis of 1336 HIV strains using statistical methods for predicting the affinity of the TBP/TATA complex, clustering into 3 visible groups was obtained (Fig. 1).

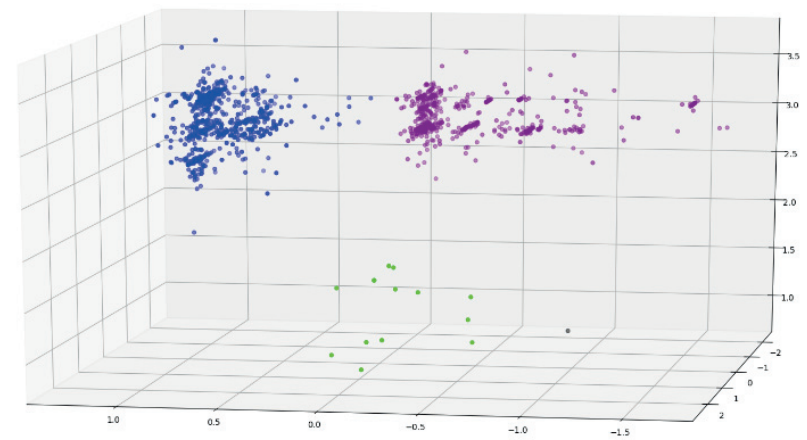

Fig. 1. Groups obtained based on the analysis of 1336 HIV strains

On the phylogenetic tree, the obtained clustering was confirmed for two groups (Fig. 2).

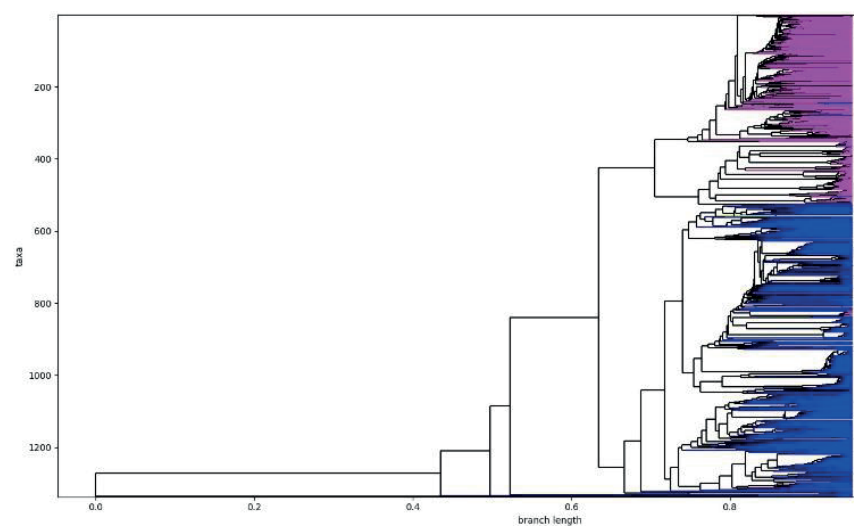

Fig. 2. The phylogenetic tree of 1336 HIV strains

In the second step, an agent computer model of the HIV epidemic was developed and implemented taking into account socio-epidemic, geographical and genetic factors.
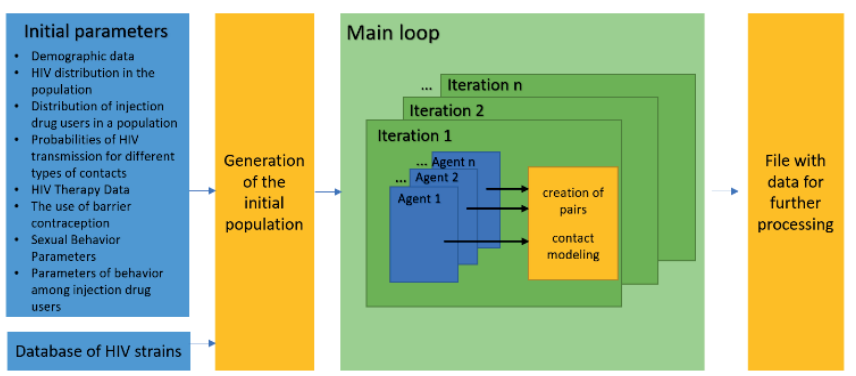

Fig. 3. HIV agent model diagram

35 strains characteristic of the territory of Russia were selected from a total of 1336 strains. Using the statistical method of computer prediction of the affinity of the TBP/TATA complex, we obtained the virulence values of these strains and integrated them into the agent model. Using 
the model, forecasts were made for a 10-year period of development of the HIV epidemic in Russia for 5 scenarios of initial parameters.

The following scenarios were considered:

1) Development of the current situation without additional measures

2) Increased use of barrier contraception + decrease in the share of joint injections

3) Increased use of barrier contraception + increased treatment coverage and decreased viral load

4) Decrease in the share of joint injections + increase in treatment coverage and decrease in viral load

5) Combining all 3 options - barrier contraception, treatment and injections.

For scenario №1, the model predicts an increase in the number of HIV-infected in the population by more than two times to $2.45 \%$. The percentage of new HIV infections per year remains stable. The most effective strategy was №5, which suggests - 1) an increase in the proportion of men and women using barrier contraception to 0.8 (instead of 0.337 for men and 0.238) 2) a decrease in the share of joint injections to 0.01 (instead of 0.4 ) and 3 ) increasing the proportion of patients using treatment to 0.9 and increasing the proportion of patients with reduced viral load among those on treatment to 0.9 (instead of 0.45 and 0.62 , respectively). This scenario showed a 3 -fold decrease in the percentage of HIV patients in the population from 0.97 to $0.3 \%$ over 10 years and an almost complete halt in the growth of new HIV cases.
Figure 4 shows the dynamics over 10 years of the representation of 35 strains selected for Russia in the population. The most common strain MH330341 is presented 2 times more often than the least represented MK984160.

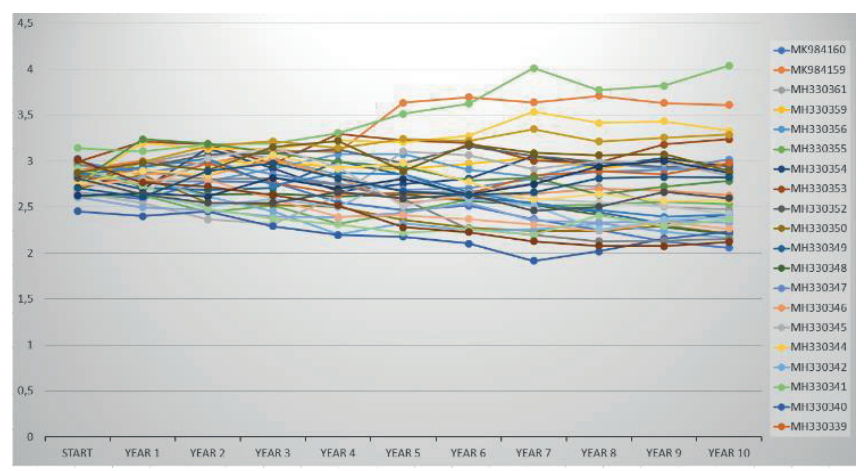

Fig. 4. The dynamics of the representation of strains in the general population over time

\section{ACKNOWLEDGMENT}

The study was supported by the Budget Project № 02592019-0008-C-01.

\section{REFERENCES}

[1] Suslov V.V., Ponomarenko P.M., Efimov V.M., Savinkova L.K., Ponomarenko M.P., Kolchanov N.A., SNPs in the HIV-1 TATA box and the AIDS pandemic. Journal of Bioinformatics and Computational Biology. 2010;8(3):607-625 